\title{
Physical Properties and Biocompatibility of a Core-Sheath Structure Composite Scaffold for Bone Tissue Engineering In Vitro
}

\author{
Chuangjian Wang, ${ }^{1}$ Guolin Meng, ${ }^{1}$ Laquan Zhang, ${ }^{2}$ Zuo Xiong, ${ }^{2}$ and Jian Liu' \\ ${ }^{1}$ Institute of Orthopaedics and Traumatology, Xijing Hospital, The Fourth Military Medical University, Xi'an 710032, China \\ ${ }^{2}$ Department of Mechanical Engineering, Tsinghua University, Beijing 100084, China
}

Correspondence should be addressed to Jian Liu, wangchuang20051983@126.com

Received 1 November 2011; Revised 17 December 2011; Accepted 4 January 2012

Academic Editor: Bernd H. A. Rehm

Copyright (C) 2012 Chuangjian Wang et al. This is an open access article distributed under the Creative Commons Attribution License, which permits unrestricted use, distribution, and reproduction in any medium, provided the original work is properly cited.

\begin{abstract}
Scaffolds play a critical role in the practical realization of bone tissue engineering. The purpose of this study was to assess whether a core-sheath structure composite scaffold possesses admirable physical properties and biocompatibility in vitro. A novel scaffold composed of poly(lactic-co-glycolic acid)/ $\beta$-tricalcium phosphate (PLGA/ $\beta$-TCP) skeleton wrapped with Type I collagen via lowtemperature deposition manufacturing (LDM) was prepared, and bone mesenchymal stem cells (BMSCs) were used to evaluate cell behavior on the scaffold. PLGA/ $\beta$-TCP skeleton was chosen as the control group. Physical properties were evaluated by pority ratio, compressive strength, and Young's modulus. Scanning electron microscope (SEM) was used to study morphology of cells. Hydrophilicity was evaluated by water absorption ratio. Cell proliferation was tested by 3-(4,5-dimethylthiazol-2-yl)-2,5-diphenyl tetrazolium bromide assay (MTT). Osteogenic differentiation of BMSCs was evaluated by alkaline phosphates activity (ALP). The results indicated that physical properties of the novel scaffold were as good as those of the control group, hydrophilicity was observably better $(P<0.01)$ than that of control group, and abilities of proliferation and osteogenic differentiation of BMSCs on novel scaffold were significantly greater $(P<0.05)$ than those of control group, which suggests that the novel scaffold possesses preferable characteristics and have high value in bone tissue engineering.
\end{abstract}

\section{Introduction}

Large bone defects, such as acute injuries, fall fractures in osteoporotic patients, or tumors and congenital malformations of the musculoskeletal system are very common in the clinical cases of orthopedics. It is necessary to resect the affected parts of the bone [1], which is a major therapeutic challenge for the reconstructive surgery after resection. Recently, great progress has been made in bone tissue engineering, which is promising for treatment of bone defects and bone regeneration [2]. Scaffold is one of the critical elements, and it is generally acknowledged that appropriate physical structure and good biocompatibility are two important characteristics that are considered ideal for bone tissue engineering. The scaffold, as a temporary template or substrate, formulates the final shape of the new bone, and the architecture of the scaffold, a key property of the scaffolds, determines its interaction with the targeted cells. Target cells behave distinctively when they grow on different scaffolds (hydrophilicity, surface roughness, etc.) [3].

In the previous study, we had fabricated 3-dimensional porous poly(lactic-co-glycolic acid) (PLGA) $/ \beta$-tricalcium phosphate $(\beta$-TCP) (PLGA/ $\beta$-TCP) scaffold via lowtemperature deposition manufacturing (LDM) [4]. In vitro and in vivo experiments had proved that the scaffold had favourable mechanical strength, high parity ratio, adjustable biodegradation rate, and facility of process and molding [5], which satisfied the essential requirements of the scaffold for the bone tissue engineering. However, the hydrophobic surface of PLGA $/ \beta$-TCP is not adequate for cell adhesion, proliferation, and osteoblastic differentiation 
$[6,7]$, which limited the repairing ability of the scaffold. Satisfactory hydrophilicity and favourable biocompatibility of the scaffold could guarantee the cells to adhere, proliferate and differentiate, and it also could promote infiltration of oxygen and nutritive material of the body fluid inside the scaffold, which is vital to the successful repair of bone defects. Therefore, it is important to modify the surface of the scaffold to achieve satisfied surface characteristics for cell adhesion, proliferation and differentiation.

Ma reported that water-absorption ratio of the porous scaffold could be improved remarkably by covering the surface of the scaffold with collagen [8]. Some studies reported that the adhesion, proliferation, and the differentiation to osteoblast directionally of the bone mesenchymal stem cells (bmscs) could be improved when the cells cultured on the type I collagen [9-11]. For these reasons, we have fabricated the core-sheath structure composite scaffold composed of $\mathrm{plga} / \beta$-tcp skeleton wrapped with type i collagen on the surface. The core-sheath structure composite scaffold was a 3-dimensional structural bone bracket stuff fabricated by a kind of controllable tachy-forming novel sprayer via $\mathrm{ldm}$. Its materials and structure of the scaffold are fairly biomimetic, and the production of the scaffold is based on the bionic principle through simulating human bone actual architecture. The aim of the present study was to analyze the physical properties and the biocompatibility of the core-sheath structure composite scaffold in comparison with $\mathrm{plga} / \beta$-tcp skeleton in vitro. Physical properties were evaluated by means of analyzing the pority ratio, aperture, compressive strength, and young's modulus. The morphology of the scaffolds and the cells on the surfaces of the scaffolds were investigated by scanning electron microscope (sem). The hydrophilicity was assessed by means of water absorption, and the proliferation of the cells were assessed by 3-(4,5-dimethylthiazol-2-yl)-2,5-diphenyl tetrazolium bromide assay (mtt). The function of the differentiated bmscs was monitored by measuring alkaline phosphates activity (alp) of the cells.

\section{Materials and Methods}

2.1. Preparation of the Materials. PLGA (LA/GA $=75 / 25$, $\mathrm{Mw}=104900, \mathrm{Mn}=92210, \mathrm{PI}=1.14)$ was purchased from Department of Medical Polymers Shandong Institute, and $\beta$-TCP $($ particle size $=1.9 \pm 0.7 \mu \mathrm{m})$ was purchased from Chemical Material Factory of Wenzhou. PLGA and $\beta$-TCP were both dissolved by 1,4-Dioxane, and the PLGA and $\beta$ TCP solution concentration was $25 \%$. Type I collagen was derived from fresh grown rats' tails following Schor's method [12] in Institute of Orthopaedics and Traumatology, Xijing Hospital, The Fourth Military Medical University. Type I collagen was dissolved by $0.2 \mathrm{~mol} / \mathrm{L}$ acetic acid, and the collagen solution concentration was $0.8 \mathrm{wt} \%$.

2.2. Fabrication of the Core-Sheath Structure Composite Scaffold. The PLGA/ $\beta$-TCP skeleton was fabricated by a LDM system as we reported before [13-16]. The core-sheath structure composite scaffold was also fabricated by a LDM system (designed by the Department of Mechanical Engineering of Tsinghua University). Briefly, PLGA, $\beta$-TCP (PLGA/ $\beta$ TCP $=7: 3$ ), and 1,4-dioxane were mixed at the room temperature to make a homogeneous slurry, and the PLGA $/ \beta$ TCP solution concentration was $25 \%$; type I collagen was dissolved by $0.2 \mathrm{~mol} / \mathrm{L}$ acetic acid at the room temperature to make a homogeneous slurry, and the collagen solution concentration was $0.8 \mathrm{wt} \%$. We prepared a drive pipe constructed with an inner pipe and an outer pipe, the terminals of which were equipped with annular tubes nozzles separately. Then the slurry of PLGA/ $\beta$-TCP and the solution of type I collagen were divided into the inner pipe and outer pipe of the drivepipe separately, and then extruded from a computer-controlled annular tubes nozzle line by line onto a platform or frozen materials in a low-temperature room simultaneously. The slurry and solution would both solidify quickly after being extruded, and, at the same time, the PLGA $/ \beta$-TCP and the type I collagen would combine tightly. Rounded macropores $(300-500 \mu \mathrm{m})$ appeared at intervals among lines. When one layer finished, the platform moved down one-layer height and started a new layer. The nozzle moved in $X-Y$ plane alternately, and the platform moved in $Z$ direction repeatedly until construction of the scaffold was completed. Then the frozen scaffold was freeze dried in a freeze dryer, through which the micropores (less than $10 \mu \mathrm{m}$ ) formed as the phase separation process happened in the lowtemperature room. Finally, the scaffold was cross-linked by carbodiimide.

2.3. SEM Observation. SEM (S-3400N; Hitachi, Tokyo, Japan) was used to observe the surface and the microstructure of the scaffolds and the interface configuration of the PLGA $/ \beta$-TCP and type I collagen. Before the observation, each sample from the two groups was freeze dried and coated with a gold layer using a sputter coater.

2.4. Test of Scaffold Pority Ratio. Pority ratio was evaluated according to the apparent densities method following Vaz et al. [17]. The samples were cut into cubes after frozen in liquid nitrogen. Their apparent volumes were calculated after measurement of the apparent dimensions of the cubes, and the samples were freeze dried in a freeze dryer for $24 \mathrm{~h}$, then the weights of the dry samples were measured to an accuracy of $10^{-4} \mathrm{~g}(n=3)$. The apparent density was calculated as follow:

$$
\rho=\frac{m}{V}
$$

The total pority ratio $P_{t}$ was calculated according to

$$
P_{t}=\left(1-\frac{\rho}{\rho_{o}}\right) \times 100
$$

where $m$ is the mass of dry samples, $V$ is the volume of the samples, $\rho$ is the apparent density of the samples, and $\rho_{o}$ is the standard density of the samples.

2.5. Assessment of Mechanical Strength. Scaffolds with the size of $6.5 \times 6.5 \times 13.5 \mathrm{~mm}^{3}$ were prepared, and material 
testing was carried out using a computer-controlled electronic universal material testing machine (Shimadzu, Kyoto, Japan) to determine the compressive strength and the Young's modulus. The samples were compressed using a standard method with speed-controlled compression force of $1 \mathrm{~mm} / \mathrm{min}$. Data were recorded every second $(n=3)$.

2.6. Water Absorption of Scaffolds. Scaffolds with the size $1 \times$ $1 \times 1 \mathrm{~cm}^{3}$ were used for water absorption test. Samples with the required size were immersed in phosphate-buffered saline (PBS, $\mathrm{pH} 7.4$ ), and the weight of each sample was recorded $\left(W_{i}\right)$. Then the PLGA/ $\beta$-TCP skeleton and the core-sheath structure composite scaffold were incubated in $500 \mathrm{~mL}$ PBS and maintained in a humidified incubator at $37^{\circ} \mathrm{C}$ for 7 days. The samples were then removed from the PBS, gently blotted with filter paper to remove surface water, and immediately weighed $\left(W_{s}\right)$. The water-absorption ratios were calculated using the following equation:

Water-absorption ratio $=\left[\frac{\left(W_{s}-W_{i}\right)}{\left(W_{s}\right)}\right] \times 100 \%$.

2.7. Culture and Seeding of BMSCs on Scaffolds. Each sample from two groups with the size $1 \times 1 \times 0.4 \mathrm{~cm}^{3}$ was sterilized with ethylene oxide sterilization method. The sterilized scaffolds were immersed into low-glucose dulbecco's modified eagle media (LG-DMEM, HyClone, Utah, USA) complete medium (containing 20\% fetal bovine serum (FBS; HyClone, Utah, USA) and 100 units $/ \mathrm{mL}$ penicillin and $100 \mu \mathrm{g} / \mathrm{mL}$ streptomycin) for $12 \mathrm{~h}$ before cell seeding. BMSCs were obtained from the femurs and tibiae of a 1-day-old New Zealand white rabbit. The BMSCs were incubated at $37^{\circ} \mathrm{C}$ in a humidified environment of $5 \% \mathrm{CO}_{2}$, in flasks containing the LG-DMEM complete medium. The medium was changed every 2-3 days. When the culture flasks became confluent, cells were detached with $0.25 \%$ trypsin (Sigma-Aldrich, St. Louis, MO, USA) and passaged. Cell subcultures of third passages were used in the experiments. Osteogenic differentiation was induced by culturing BMSCs in osteogenic medium (DMEM supplemented with $10 \%$ FBS, $10^{-8} \mathrm{~mol} / \mathrm{L}$ dexamethasone, $10 \mathrm{mM} \beta$-glycerol phosphate, and $50 \mathrm{mg} / \mathrm{mL}$ L-ascorbic acid) (Sigma-Aldrich, St. Louis, MO, USA) for 3 weeks. Then, the cells were digested from the culture flasks by incubation with $0.25 \%$ trypsin, centrifuged for $5 \mathrm{~min}$ at $1000 \mathrm{rpm}$, resuspended with LGDMEM medium seeded onto the top of the scaffolds, and placed in a polystyrene 6-well plate, and incubated at $37^{\circ} \mathrm{C}$ to allow the full adhesion of cells to the scaffolds. Cell culture was performed with medium changed every 2-3 days.

2.8. BMSCs Proliferation Assay and SEM Observation. The proliferation of BMSCs was detected by MTT. Briefly, $250 \mu \mathrm{L}$ of the cells suspension were seeded at each scaffold in 6well plates at a cellular density of $1 \times 10^{7}$ cells $/ \mathrm{mL}$ for the cell proliferation assay. After $4 \mathrm{~h}, 2 \mathrm{~d}, 6 \mathrm{~d}, 10 \mathrm{~d}$, and $14 \mathrm{~d}$ of culturing, the proliferation state of the BMSCs on the surfaces was analyzed by MTT at OD $490 \mathrm{~nm}(n=3)$.
The morphologies of MSCs seeded on the scaffolds after culture for $4 \mathrm{~h}, 2 \mathrm{~d}, 6 \mathrm{~d}$, and $14 \mathrm{~d}$ were observed by SEM. The samples were taken out of the culture plates, washed with PBS three times, and fixed in 3\% glutaraldehyde in $\mathrm{pH}$ 7.4 PBS for $24 \mathrm{~h}$ at $4^{\circ} \mathrm{C}$, and the cells were then rinsed in PBS twice, postfixed in $1 \%$ osmium tetroxide, dehydrated in a graded series of alcohol and subsequently critical point dried, and then sputter coated with a thin layer of gold for observation under the scanning electron microscope operated at $5 \mathrm{kv}$.

2.9. ALP Assay. The relative ALP activity was determined according to colorimetric method with p-nitrophenyl as the substrate [18]. Briefly, the cells in both groups were collected as before on $6 \mathrm{~d}, 10 \mathrm{~d}$, and $14 \mathrm{~d}$. Then the cell pellets were rinsed with PBS for three times and lysed with sonication. Sample volumes of $0.1 \mathrm{~mL}$ were mixed with $0.1 \mathrm{~mL}$ p-nitrophenyl phosphate (pNPP) in $0.1 \mathrm{M}$ glycine ( $\mathrm{pH}$ 13.0), and the mixture was incubated at $37^{\circ} \mathrm{C}$ for $30 \mathrm{~min}$ on a bench shaker. The enzymatic reaction was stopped by the addition of $0.3 \mathrm{~mL}$ of $0.25 \mathrm{~N} \mathrm{NaOH}$. Enzyme activity was quantified by absorbance measurements at $405 \mathrm{~nm}$ for the amount of p-nitrophenol (Sigma-Aldrich, St. Louis, MO, USA) liberated. Similarly, nonseeded biomaterials were prepared in an identical manner and analyzed as blank controls.

2.10. Statistical Evaluation. The number of independent replica is listed individually for each experiment. All data are expressed as mean value \pm standard deviation and analysed by SPSS 13.0, and $t$ test was used for hypothesis testing. Statistically significant values are defined as $P<0.05$.

\section{Results}

3.1. Morphology of the Core-Sheath Structure Composite Scaffold. Figures 1(a), 1(c), and 1(e) and Figures 1(b), 1(d), and $1(\mathrm{f})$ are the magnified image of the side face of the PLGA $/ \beta$ TCP skeletons and the core-sheath structure composite scaffolds. Compared with the PLGA/ $\beta$-TCP skeleton, the surface of the construction unit of the core-sheath structure composite scaffold is totally wrapped by the type I collagen. Figure $1(\mathrm{~g})$ shows that the interface of the PLGA/ $\beta$-TCP and type I collagen is combined tightly.

\subsection{Pority Ratio and Aperture of the Core-Sheath Structure} Composite Scaffold. Table 1 shows that the pority ratio of the PLGA/ $\beta$-TCP skeleton and the core-sheath structure composite scaffold are $88.1 \pm 1.2$ and $86.7 \pm 3.6$, respectively, and the statistical analysis demonstrated that there was no significant difference in the pority ratio of the two scaffolds. Figures 1(a) and 1(b) illustrate that the macropore dimensions of the two scaffolds are both $300-500 \mu \mathrm{m}$ in size.

3.3. Mechanical Strength of the Core-Sheath Structure Composite Scaffold. The compressive strength of the PLGA/ $\beta$ TCP skeleton and the core-sheath structure composite scaffold are $0.68 \pm 0.04$ and $0.70 \pm 0.07$, and the Young's 


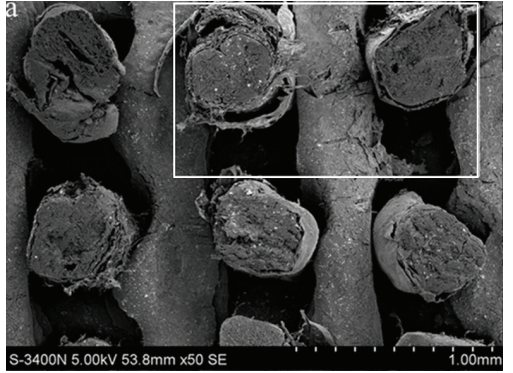

(a)

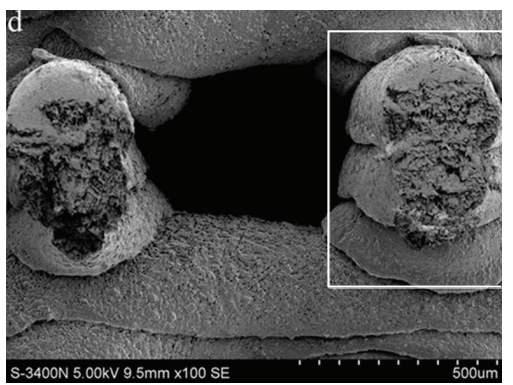

(d)

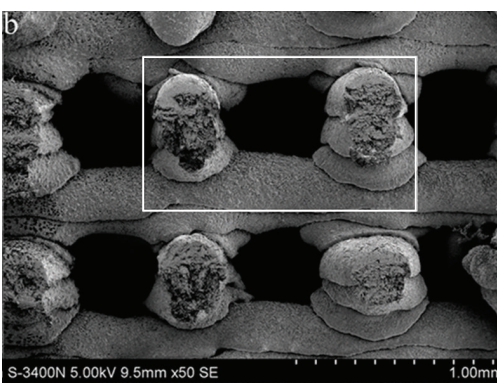

(b)

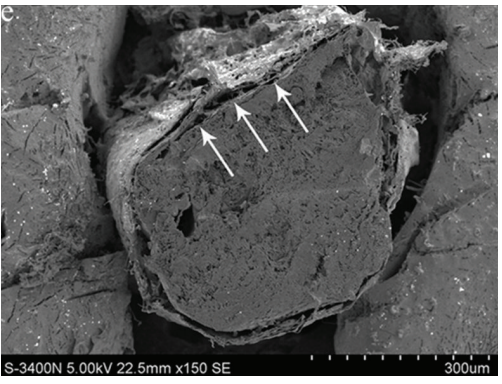

(e)

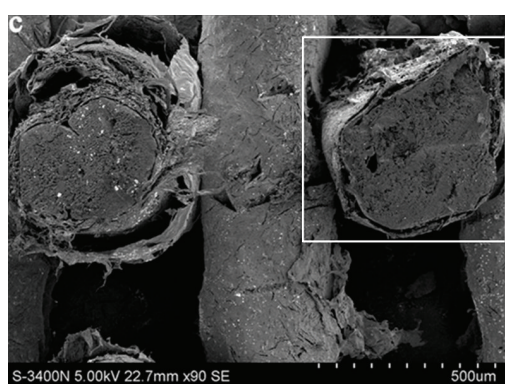

(c)

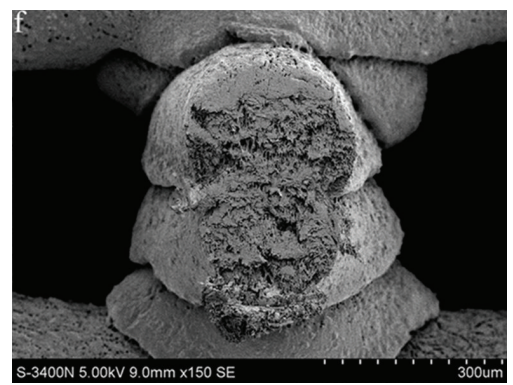

(f)

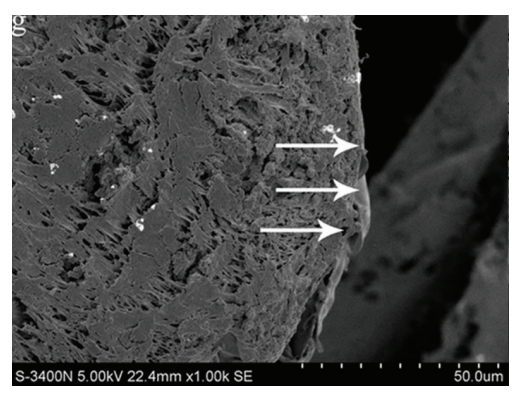

(g)

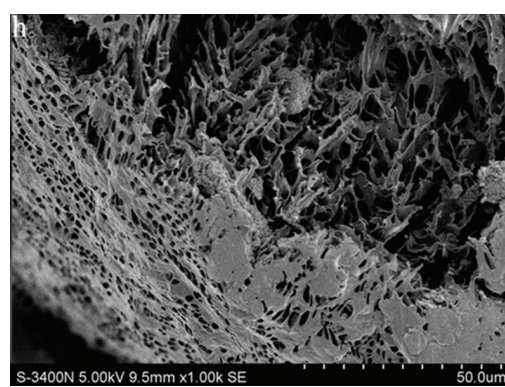

(h)

FIGURE 1: ( $\mathrm{a}$ and $\mathrm{b}$ ) Morphological comparison observation by SEM of the core-sheath structure composite scaffold and the PLGA/ $\beta$-TCP skeleton $(\times 50,50)$. (c and e) Magnified view of the white rectangle frame from $(\mathrm{a})(\times 90,150)$. Note: the arrows point to the collagen wrapped the PLGA/ $\beta$-TCP. ( $\mathrm{d}$ and $\mathrm{f}$ ) Magnified view of the white rectangle frame from (b) $(\times 100,150)$. (g) Morphological observation by SEM of the core-sheath structure composite scaffold $(\times 1 \mathrm{k})$. Note: the arrows point to the interface of the PLGA/ $\beta$-TCP and the collagen. (h) Morphological observation by SEM of the PLGA/ $\beta$-TCP skeleton. $(\times 1 \mathrm{k})$.

TABLE 1: Water-absorption ratio, pority ratio, and mechanical strength of the PLGA/ $\beta$-TCP scaffolds and the core-sheath structure composite scaffolds.

\begin{tabular}{lcccc}
\hline Material & Water-absorption ratio $(\%)$ & Parity ratio $(\%)$ & Compressive strength (Mpa) & Young's modulus (Mpa) \\
\hline PLGA/ $\beta$-TCP & $2.8 \pm 0.2$ & $88.1 \pm 1.2$ & $0.68 \pm 0.04$ & $17.91 \pm 2.12$ \\
core-sheath & $16.1 \pm 0.3^{*}$ & $86.7 \pm 3.6$ & $0.70 \pm 0.07$ & $18.16 \pm 3.21$ \\
\hline
\end{tabular}

${ }^{*}$ Significantly different compared with the PLGA/ $\beta$-TCP scaffolds $(n=3, P<0.01)$.

modulus are $17.91 \pm 2.12$ and $18.16 \pm 3.21$, respectively Table 1. The results indicated that there was no significant difference between the two scaffolds in both the compressive strength and the Young's modulus.

3.4. Hydrophilicity of the Core-Sheath Structure Composite Scaffold. The water-absorption capacity of the PLGA/ $\beta$ TCP skeleton is $2.8 \pm 0.2 \%$, and the core-sheath structure composite scaffold is $16.1 \pm 0.3 \%$ Table 1 . The statistical analysis demonstrated that there was observable difference. $(P<0.01)(n=3)$.

3.5. Adhesion and Proliferation of MSCs on the Core-Sheath Structure Composite Scaffold. The result of MTT indicated that the number of BMSCs in the core-sheath structure composite scaffolds was greater significantly than that of the 


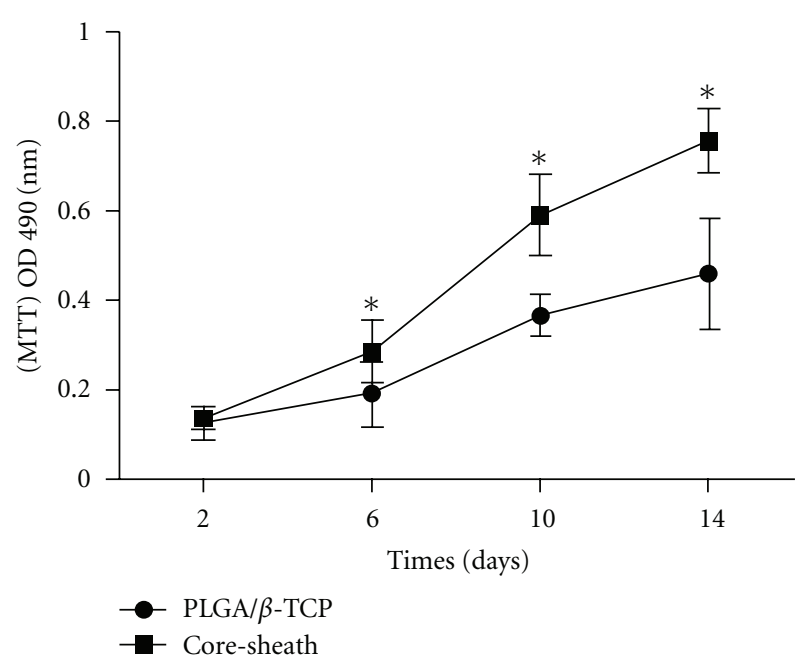

FIGURE 2: Cell proliferation comparisons on the core-sheath structure composite scaffold and the PLGA/ $\beta$-TCP skeleton. Cell proliferation analysis by MTT methods. Each value and error bar represent the mean of triplicate samples and their standard deviation $\left({ }^{*} P<0.05\right.$ compared with the PLGA $\beta$-TCP scaffolds; $n=3)$.

PLGA/ $\beta$-TCP skeleton at different intervals from day $6(P<$ $0.05)$, and the number of BMSCs in two kinds of scaffolds was both increased from day 2 to day 14 Figure 2.

On hour 4, the differentiated BMSCs adhered to the coresheath structure composite scaffold and the pseudopodium of the cells stretched out Figure 3(a). At day 2, the cells stretched onto the core-sheath structure composite scaffold and the pseudopodium of the cells tightly attached to the surface of the scaffold Figures 3(c) and 3(d). At day 6, the cells clustered on the core-sheath structure composite scaffold and were abounded with pseudopodium Figures $3(\mathrm{~g})$ and $3(\mathrm{~h})$. At day 14, the cells covered the surface of the porous core-sheath structure composite scaffold or extended toward the pores Figures $3(\mathrm{~m})$ and $3(\mathrm{n})$, what's more, the BMSCs secreted ECM Figures $3(\mathrm{k})$ and $3(\mathrm{l})$. Obviously, there were fewer cells adhering to the PLGA/ $\beta$ TCP skeleton proliferation compared with the core-sheath structure composite scaffold Figures 3(b), 3(e), 3(f), 3(i), $3(\mathrm{j}), 3(\mathrm{o})$ and $3(\mathrm{p})$.

3.6. Osteoblastic Differentiation of MSCs on the Core-Sheath Structure Composite Scaffold. Figure 4 shows that the ALPase expression on each scaffold is increased substantially from day 6 to day 14, and significantly higher activities on 6,10 , and 14 days of cultivation on the core-sheath structure composite scaffolds are observed as compared with the control groups $(P<0.05)$.

\section{Discussion}

In the present study, we analyzed a core-sheath structure composite scaffold for bone tissue engineering applications. Such a scaffold may be particularly suited for implantation into bone defects due to its admirable physical properties and appropriate biocompatibility.

Ideal scaffolds for bone tissue engineering should have appropriate 3-dimensional porous structure, favourable mechanical strength, adjustable degradation rate, and facility of process and molding. However, it is practically impossible to satisfy the numerous requirements for scaffold materials by using a single material; therefore, composite systems, which combine the advantages of different materials, are becoming more and more promising. One class of such composites comprises polymers-inorganic materials which come up to those standards [2]. The materials we chose for the composite scaffold were synthetic polymers PLGA and inorganic material $\beta$-TCP. But hydrophobicity of the composite material's surface counts against the adhesion and proliferation of the cells $[6,7]$. The hydrophilicity of the scaffold's surface is one of the important factors that facilitate the penetration of nutrients into the scaffold and the exudation of the metabolic products, which thus guaranteeing the nutrition transport and cell growth inside the scaffold [19]. It is acknowledged that material surface will contact and interact with the cells first, so the superficial physicochemical property of the material such as hydrophilicity or hydrophobicity can influence a series of the cells' responses: adhesion, proliferation, differentiation, and so forth. A lot of studies have authenticated that extracellular matrix (ECM) plays a very important part in the adhesion, proliferation, and differentiation of the cells in the surface of the materials, and type I collagen containing tropocollagen molecule is the basis of the ECM and possesses favourable hydrophilicity and low immunogenicity. So it would probably improve the superficial hydrophilicity of the composite material effectively if the surface of the composite material is wrapped with type I collagen. Therefore, we chose PLGA, $\beta$-TCP, and type I collagen as the raw and processing materials and fabricated the core-sheath structure composite scaffold so as to improve the hydrophilicity of the scaffold.

From Figure 1, we can see that the PLGA/ $\beta$-TCP is totally wrapped toroidally by type I collagen and that the covering layer is well distributed. Figure $1(\mathrm{~g})$ indicates that the contact surface of PLGA/ $\beta$-TCP and type I collagen is tight. The cementation of the PLGA/ $\beta$-TCP and type I collagen mainly depends on the diffusion action: the PLGA/ $\beta$-TCP solution (PLGA/ $\beta$-TCP dissolved in the solvent 1,4 -dioxane) and the type I collagen solution (type I collagen dissolved in the solvent $2 \%$ acetic acid) contacted each other in the process of the molding, and the 1,4-dioxane and 2\% acetic acid could mutually be dissolved very well, and the sizes of the two solvents were far less than the two solutes (macromolecule chain), so the two solutions could interdiffuse quickly and smoothly with each other. Both of the PLGA/ $\beta$-TCP molecular chain and the type I collagen polypeptide chain diffused to the contact surface, and then solidified, and the colliquefaction part was shaped, producing the joint part of the PLGA/ $\beta$-TCP and type I collagen. So, the bonding force of the two materials was strengthened.

Appropriate pority ratio and pore size are crucial to bone tissue engineering, which can produce a biological environment conducive to adhesion, proliferation of cells, 


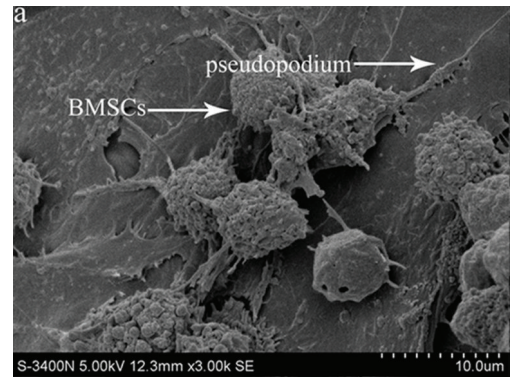

(a)

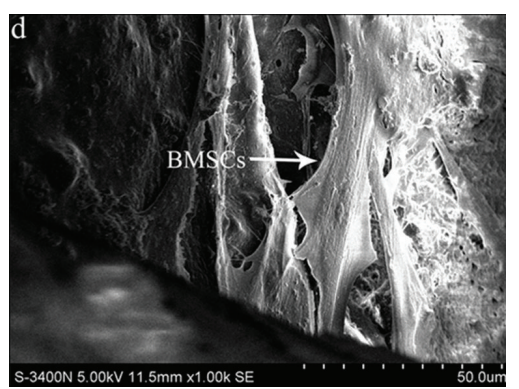

(d)

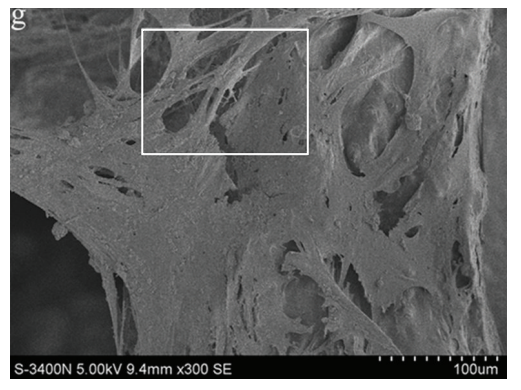

(g)

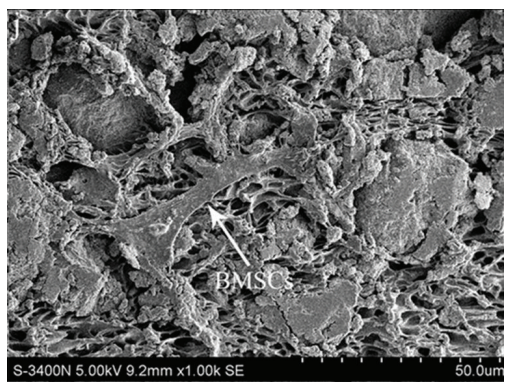

(j)

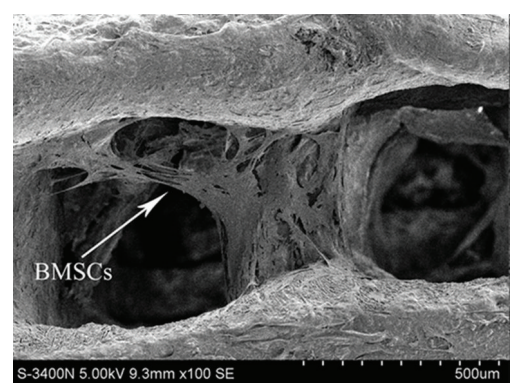

(m)

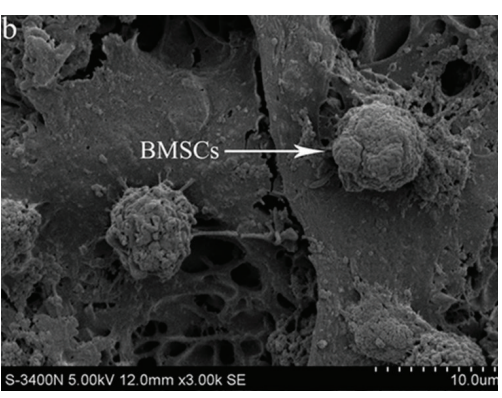

(b)

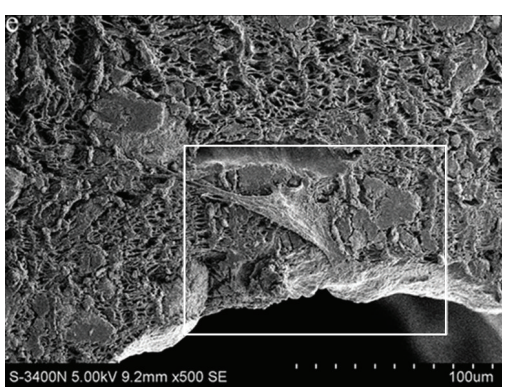

(e)

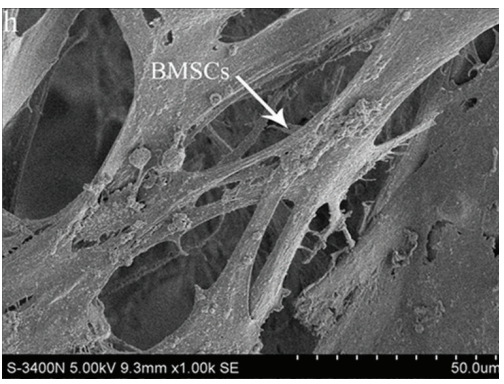

(h)

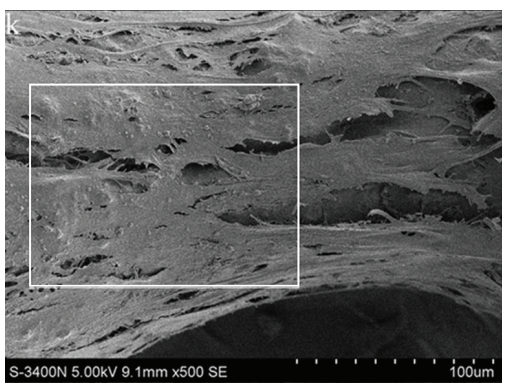

(k)

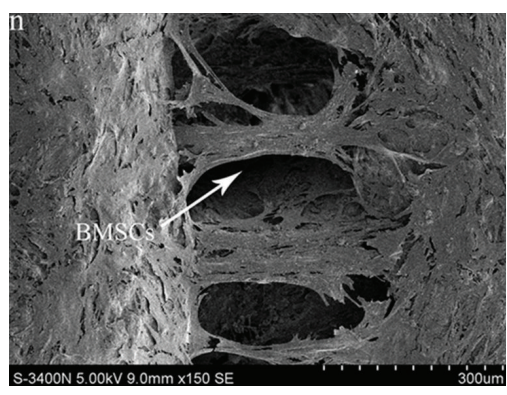

(n)

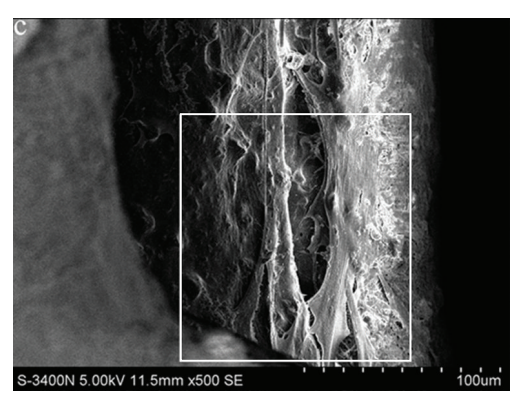

(c)

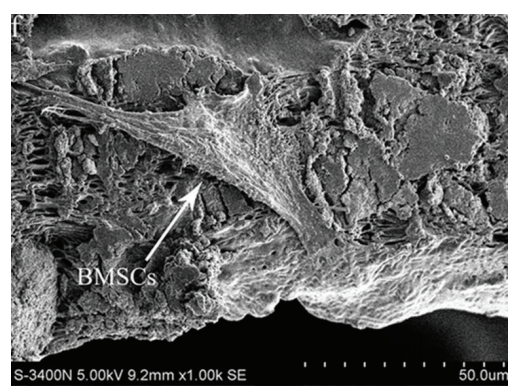

(f)

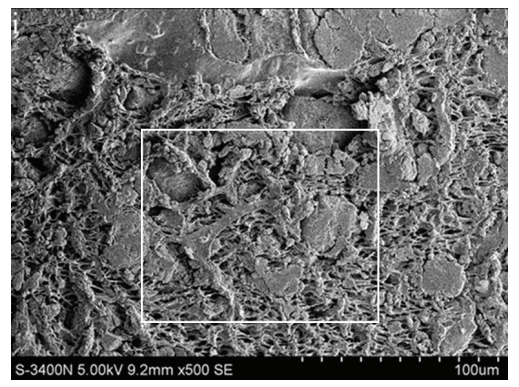

(i)

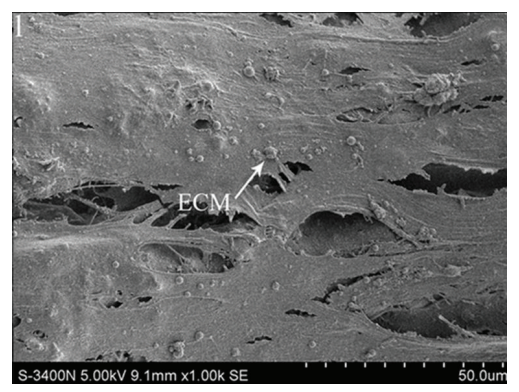

(1)

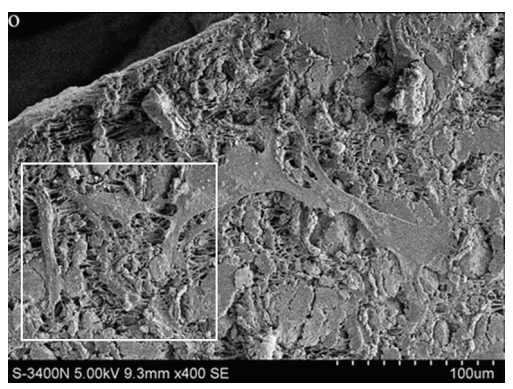

(o)

FIgUre 3: Continued. 


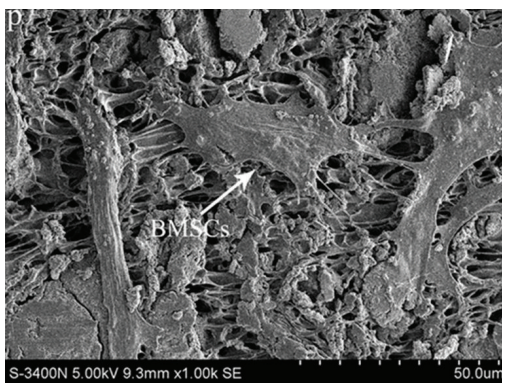

$(\mathrm{p})$

Figure 3: (a) Morphological observation of BMSCs cultured on the core-sheath structure composite scaffold by SEM after 4 hours $(\times 3.0 \mathrm{k})$. Note: the arrows point to the BMSCs adhered on the scaffold and the pseudopodium of the cells. (c and d) Morphological observation of BMSCs cultured on the core-sheath structure composite scaffold by SEM after 2 days $(\times 500,1000)$. Note: the arrows point to the BMSCs stretched onto the scaffold. ( $g$ and h) Morphological observation of BMSCs cultured on the core-sheath structure composite scaffold by SEM after 6 days $(\times 300,1000)$. Note: the arrows point to the BMSCs. $(\mathrm{k}, 1, \mathrm{~m}$, and $\mathrm{n})$ Morphological observation of BMSCs cultured on the core-sheath structure composite scaffold by SEM after 14 days $(\times 500,1000)$. Note: $(\mathrm{k}$ and $\mathrm{l})$ the arrows point to the BMSCs and secreted ECM by BMSCs. ( $\mathrm{m}$ and $\mathrm{n}$ ) the arrows point to the BMSCs growed into the holes of the scaffold. (b) Morphological observation of BMSCs cultured on the PLGA $\beta$-TCP skeleton after 4 hours $(\times 3.0 \mathrm{k}$ ). (e and $\mathrm{f})$ Morphological observation of BMSCs cultured on the PLGA/ $\beta$-TCP skeleton after 2 days $(\times 500,1000)$. Note: the arrows point to the BMSCs. (I and j) Morphological observation of BMSCs cultured on the PLGA/ $\beta$-TCP skeleton after 6 days $(\times 500,1000)$. Note: the arrows point to the BMSCs. (o and p) Morphological observation of BMSCs cultured on the PLGA $\beta$-TCP skeleton after 14 days $(\times 400,1000)$. Note: the arrows point to the BMSCs.

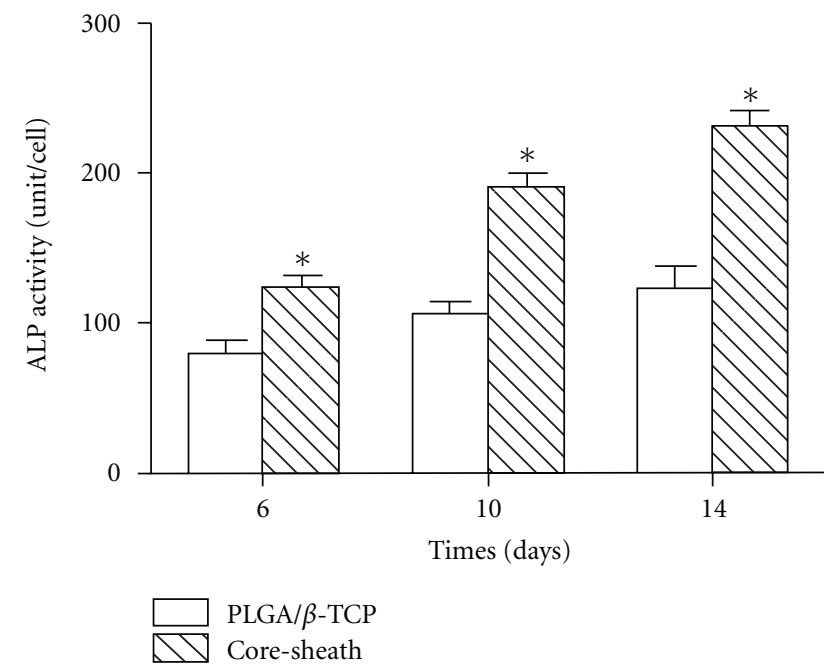

FIGURE 4: ALP activity comparisons on the core-sheath structure composite scaffold and the PLGA/ $\beta$-TCP skeleton. Each value and error bar represent the mean of triplicate samples and their standard deviation $\left({ }^{*} P<0.05\right.$ compared with the PLGA $\beta$-TCP scaffolds; $n=3)$.

and the smooth transport of nutrients and metabolic waste $[14,20]$. These properties are also necessary for bone tissue formation because migration and proliferation of osteoblasts and mesenchymal cells as well as vascularization could be allowed [10]. Results of this study indicate that both scaffolds have fairly high pority ratio (over $75 \%$ ), which is considered to be beneficial to cell growth and survival [21]. The pore size should be large enough to support cell migration and bone ingrowth such that cell coverage, pore bridging formation and occlusion can be prevented. The optimal pore size required for bone ingrowth has been suggested by some researchers to be up to $200 \mu \mathrm{m}$ [12]. The macropore dimensions of the novel scaffold are $300-500 \mu \mathrm{m}$ in size, which indicates that the scaffolds have the proper pore size for bone tissue engineering.

The values of compressive strength and Young's modulus of the two kinds of scaffolds are both lower than those of healthy bone [8]. However, varieties of orthopedic fixations have been found to be widely used in clinic, which could ensure the fastness of the fixation during bone regeneration and degradation of the artificial bone. Therefore, as a regenerative scaffold, its basic mechanical strength could be satisfied by the embedded artificial bone if only it can hold its shape when compressed by the surrounding soft tissue. In our experiments, the core-sheath structure composite scaffolds had a compressive modulus of $0.70 \pm 0.07 \mathrm{MPa}$, which meets the above standard very well.

On hour 4, the BMSCs had adhered to the novel scaffold and the pseudopodium of the cells had begun to stretch out; obviously, the BMSCs had also adhered to the PLGA/ $\beta$-TCP skeleton however, there were fewer cells adhering to it Figures 3 (a) and 3(b); from day 2 to day 14, the BMSCs stretched onto the core-sheath structure composite scaffold, and the pseudopodium of the cells tightly attached to the surface of the scaffold, and then the cells proliferated and clustered on the scaffold, furthermore, at day 14, the cells had extended into the pores, in addition, and after the scaffolds and the cells were cultivated together for 14 days, we observed the cells attached with the scaffold had secreted ECM; obviously, there were fewer proliferation of the cells on the PLGA $/ \beta$ TCP skeleton Figures $3(\mathrm{c})-3(\mathrm{p})$. From the SEM observation, we can conclude that the core-sheath structure composite scaffold had better biocompatibility, which is an important 
principle property of scaffolds and is considered ideal for bone tissue engineering. The results of MTT also indicated that the BMSCs showed better adhesion and proliferation activity on the surface of the core-sheath structure composite scaffold than on the PLGA/ $\beta$-TCP skeleton, which were consistent with the results of the SEM observation.

There are two important pillars in the bone tissue engineering field, biomaterials and cells. The former must permit the rapid grow and proliferation of the seeded cells while keep their potential for further differentiation, which is one of the key processes for bone regeneration. ALP is a kind of cell surface glycoprotein, which is involved in mineralization [22] and is the most widely recognized marker of osteoblastic differentiation [23]. According to the results, the core-sheath structure composite scaffolds possessed a greater ability to promote the osteoblastic differentiation of BMSCs than that of the PLGA/ $\beta$-TCP skeletons, which also demonstrate that the core-sheath structure composite scaffold has comparatively good biocompatibility.

Adhesion of cells on the scaffolds is the foundation of the bone tissue engineering. Adhesion of cells on the scaffolds and cells is very complex and is influenced by various factors. Surface properties of the scaffold such as electrical characteristics, free energy, hydrophilicity, or hydrophobicity are crucial to the cell adhesion, because the real touch between the scaffold and cells occurs between the material surface and the cell. The results of our study demonstrated that the hydrophilicity of the core-sheath structure composite scaffold was improved significantly, and it is acknowledged that favourable hydrophilicity is beneficial to cell adhesion [24]. Cells contacting the surface of the scaffolds were the first to attach and adhere; the quality of this adhesion would influence their morphology, and their capacity for proliferation and differentiation. The coresheath structure composite scaffold's surface was covered with type I collagen, which could improve the hydrophilicity of the scaffold, and was probably the reason for the better cell adhesion, proliferation activity, and differentiation. The results of our present study indicated that the novel scaffold was more suitable for adhesion, proliferation, and differentiation of the BMSCs than the PLGA/ $\beta$-TCP skeleton, which that demonstrated that the core-sheath structure composite scaffold possessed good biocompatibility.

\section{Conclusions}

In this study, we created a core-sheath structure composite scaffold with an annular tubes nozzle by the LDM system. The scaffold has an open pore structure with the pore size up to $300 \mu \mathrm{m}$, and over $75 \%$ of the appropriate pority ratios have been achieved. The porous scaffold has displayed proper physical properties. Moreover, the scaffold has achieved a better hydrophilicity, and BMSCs have adhered, spread, proliferated, and osteogenically differentiated well on the scaffolds. So, we may conclude that such a scaffold may act as an ideal implant into bone defects thanks to its favourable physical properties and biocompatibility. All these data may provide further proofs that the core-sheath structure composite scaffold has potential in bone tissue engineering.

\section{References}

[1] C. F. Amstein and P. A. Hartman, "Adaptation of plastic surfaces for tissue culture by glow discharge," Journal of Clinical Microbiology, vol. 2, no. 1, pp. 46-54, 1975.

[2] S. Shi, X. H. Wang, G. Guo, M. Fan, M. J. Huang, and Z. Y. Qian, "Preparation and characterization of microporous poly(D,L-lactic acid) film for tissue engineering scaffold," International Journal of Nanomedicine, vol. 5, no. 1, pp. 10491055, 2010.

[3] T. Angwarawong, S. T. Dubas, M. Arksornnukit, and P. Pavasant, "Differentiation of MC3T3-E1 on poly(4-styrenesulfonic acid-co-maleic acid) sodium salt-coated films," Dental Materials Journal, vol. 30, no. 2, pp. 158-169, 2011.

[4] C. Gao, X. Hu, Y. Hong, J. Guan, and J. Shen, "Photografting of poly(hydroxylethyl acrylate) onto porous polyurethane scaffolds to improve their endothelial cell compatibility," Journal of Biomaterials Science, Polymer Edition, vol. 14, no. 9, pp. 937-950, 2003.

[5] J. Gao, L. Niklason, and R. Langer, "Surface hydrolysis of poly(glycolic acid) meshes increases the seeding density of vascular smooth muscle cells," Journal of Biomedical Materials Research, vol. 42, no. 3, pp. 417-424, 1998.

[6] C. Y. Yang, L. Y. Huang, T. L. Shen, and J. A. Yeh, "Cell adhesion, morphology and biochemistry on nano-topographic oxidized silicon surfaces," European Cells \& Materials, vol. 20, pp. 415-430, 2010.

[7] N. M. Coelho, C. González-García, J. A. Planell, M. SalmerónSánchez, and G. Altankov, "Different assembly of type IV collagen on hydrophilic and hydrophobic substrata alters endothelial cells interaction," European Cells \& Materials, vol. 19, pp. 262-272, 2010.

[8] L. J. Gibson, "The mechanical behaviour of cancellous bone," Journal of Biomechanics, vol. 18, no. 5, pp. 317-328, 1985.

[9] M. D. Schofer, A. Veltum, C. Theisen et al., "Functionalisation of PLLA nanofiber scaffolds using a possible cooperative effect between collagen type i and BMP-2: impact on growth and osteogenic differentiation of human mesenchymal stem cells," Journal of Materials Science: Materials in Medicine, vol. 22, no. 7, pp. 1753-1762, 2011.

[10] V. Karageorgiou and D. Kaplan, "Porosity of 3D biomaterial scaffolds and osteogenesis," Biomaterials, vol. 26, no. 27, pp. 5474-5491, 2005.

[11] Y. Hatakeyama, J. Hatakeyama, A. Takahashi et al., "The effect of valproic acid on mesenchymal pluripotent cell proliferation and differentiation in extracellular matrices," Drug Target Insights, vol. 5, pp. 1-9, 2011.

[12] J. M. Lane and M. P. Bostrom, "Bone grafting and new composite biosynthetic graft materials," Instructional course lectures, vol. 47, pp. 525-534, 1998.

[13] X. Liu and P. X. Ma, "Polymeric scaffolds for bone tissue engineering," Annals of Biomedical Engineering, vol. 32, no. 3, pp. 477-486, 2004.

[14] Z. Li, H. R. Ramay, K. D. Hauch, D. Xiao, and M. Zhang, "Chitosan-alginate hybrid scaffolds for bone tissue engineering," Biomaterials, vol. 26, no. 18, pp. 3919-3928, 2005.

[15] L. Ma, Y. Shi, Y. Chen, H. Zhao, C. Gao, and C. Han, "In vitro and in vivo biological performance of collagen-chitosan/siliconemembrane bilayer dermal equivalent," 
Journal of Materials Science: Materials in Medicine, vol. 18, no. 11, pp. 2185-2191, 2007.

[16] R. Marom, I. Shur, R. Solomon, and D. Benayahu, "Characterization of adhesion and differentiation markers of osteogenic marrow stromal cells," Journal of Cellular Physiology, vol. 202, no. 1, pp. 41-48, 2005.

[17] C. M. Vaz, S. van Tuijl, C. V. C. Bouten, and F. P. T. Baaijens, "Design of scaffolds for blood vessel tissue engineering using a multi-layering electrospinning technique," Acta Biomaterialia, vol. 1, no. 5, pp. 575-582, 2005.

[18] A. M. Moursi, R. K. Globus, and C. H. Damsky, "Interactions between integrin receptors and fibronectin are required for calvarial osteoblast differentiation in vitro," Journal of Cell Science, vol. 110, no. 18, pp. 2187-2196, 1997.

[19] E. Biazar, M. Heidari, A. Asefnejad, and N. Montazeri, "The relationship between cellular adhesion and surface roughness in polystyrene modified by microwave plasma radiation," International Journal of Nanomedicine, vol. 6, pp. 631-639, 2011.

[20] T. S.N. Silva, B. T. Primo, A. N. Silva Jr., D. C. Machado, C. Viezzer, and L. A. Santos, "Use of calcium phosphate cement scaffolds for bone tissue engineering: in vitro study," Acta Cirurgica Brasileira, vol. 26, no. 1, pp. 7-11, 2011.

[21] M. De Barros Coelho and M. Magalhães Pereira, "Sol-gel synthesis of bioactive glass scaffolds for tissue engineering: effect of surfactant type and concentration," Journal of Biomedical Materials Research. Part B, vol. 75, no. 2, pp. 451-456, 2005.

[22] X. H. Xie, X. L. Wang, G. Zhang et al., "Structural and degradation characteristics of an innovative porous PLGA/TCP scaffold incorporated with bioactive molecular icaritin," Biomedical Materials, vol. 5, no. 5, Article ID 054109, 2010.

[23] Y. Zhou, F. Chen, S. T. Ho, M. A. Woodruff, T. M. Lim, and D. W. Hutmacher, "Combined marrow stromal cellsheet techniques and high-strength biodegradable composite scaffolds for engineered functional bone grafts," Biomaterials, vol. 28, no. 5, pp. 814-824, 2007.

[24] J. Ruan, K. Wang, H. Song, X. Xu, J. Ji, and D. Cui, "Biocompatibility of hydrophilic silica-coated CdTe quantum dots and magnetic nanoparticles," Nanoscale Research Letters, vol. 6, no. 1, pp. X1-13, 2011. 


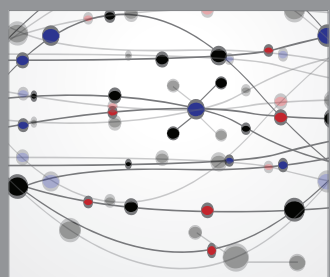

The Scientific World Journal
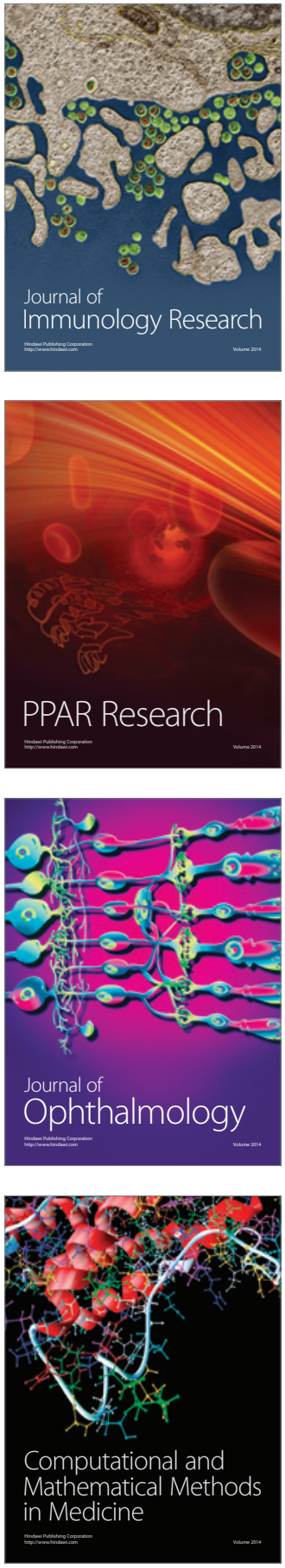

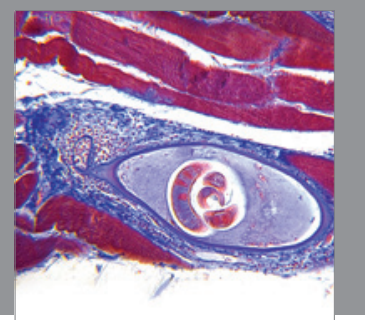

Gastroenterology

Research and Practice
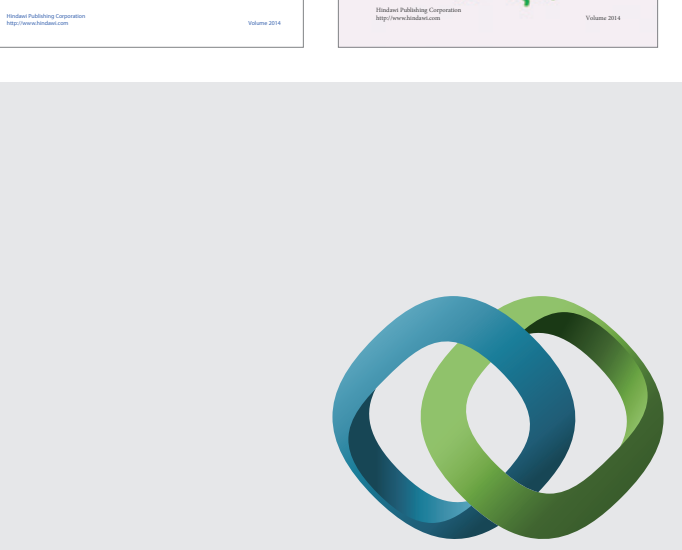

\section{Hindawi}

Submit your manuscripts at

http://www.hindawi.com
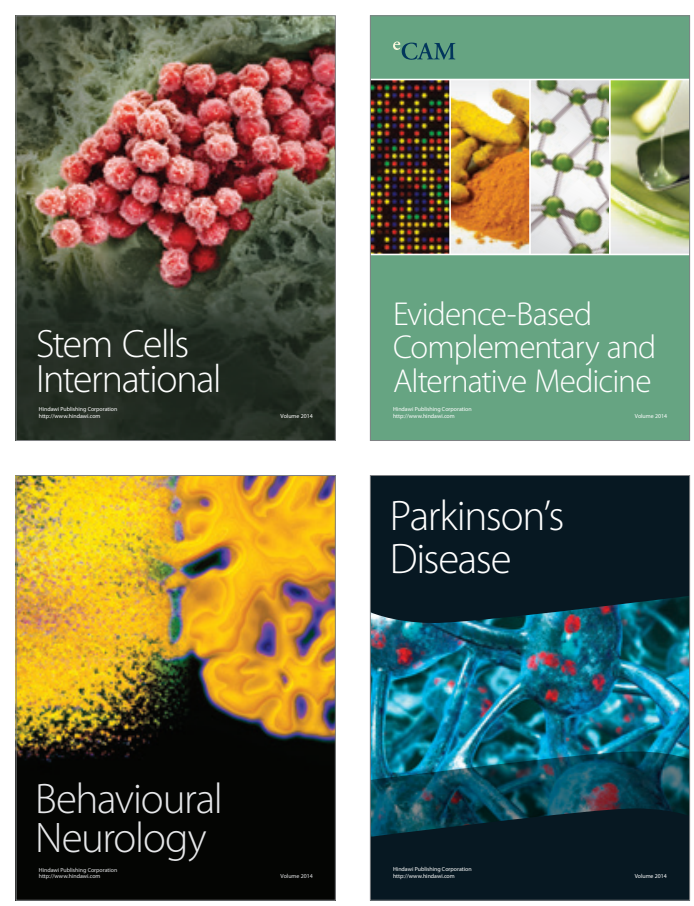

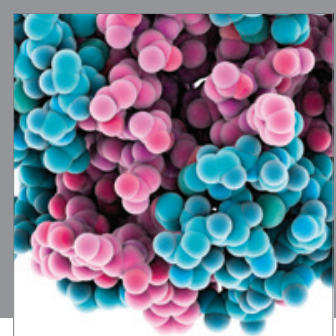

Journal of
Diabetes Research

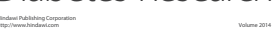

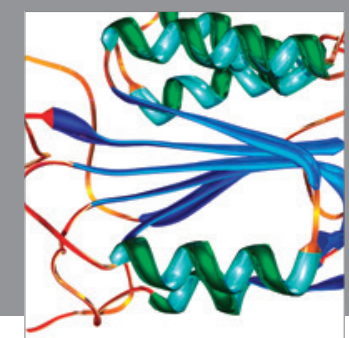

Disease Markers
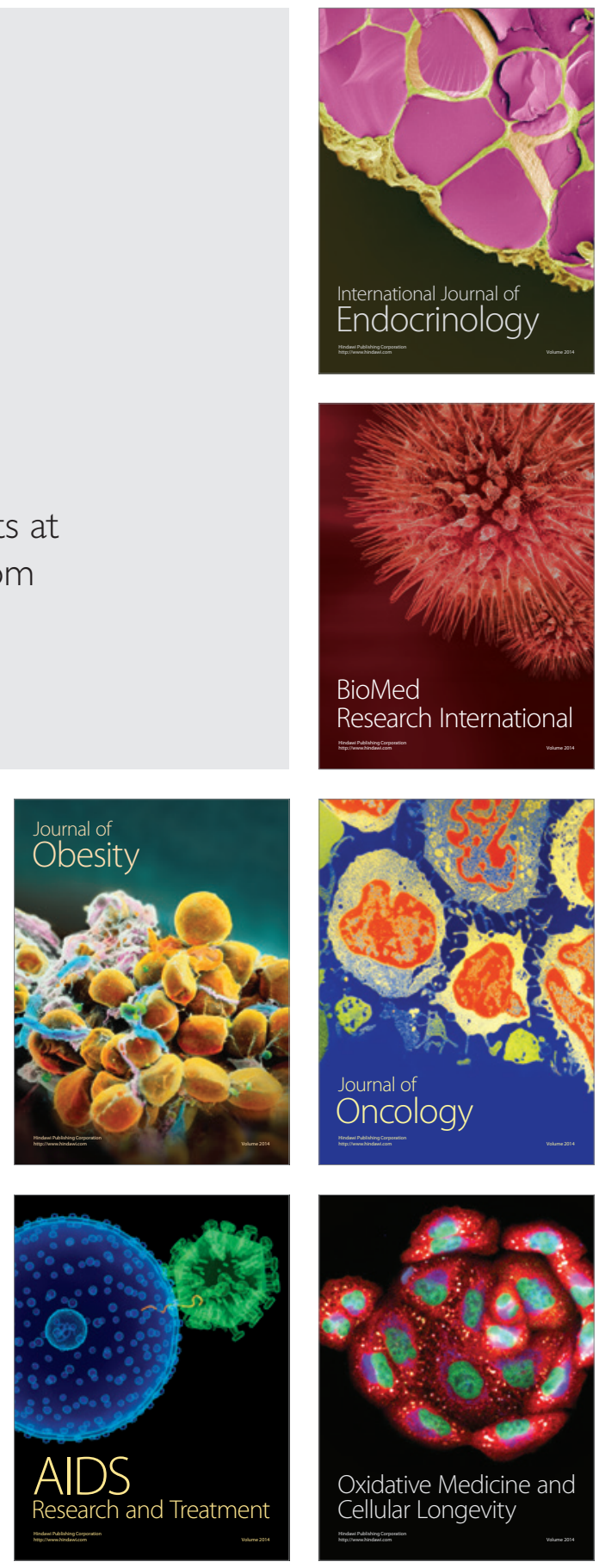